DOI : $10.22199 / \mathrm{S} 07160917.1982 .0002 .00005$

\title{
ENTREVISTA AL DR. JURGEN WEYER
}

Invitado por el Departamento de Matemáticas, visitó nuestra Universidad, el Dr. Jurgen Weyer, asesor Académico de la Embajada de Alemania Federal e Investigador de la línea de Análisis Numérico, de la Universidad de Santiago.

Durante su visita el Doctor Weyer participó en las Primeras Jornadas de Matemáticas organizadas por el Departamento de Matemáticas de la Universidad del Norte y la Sociedad de Matemática de Chile.

Entre las actividades más relevantes que realizó destacan: Charla Inaugural de la Primera Escuela de Matemática y el Cursillo "Introducción a la Optimización Convexa" desarrollado en dicha Escuela.

Concluída su participación el Dr. Weyer gentilmente accedió a una reunión con todos los Académicos de nuestro 
Departamento, donde expresó sus ideas sobre interesantes aspectos del quehacer matemático. Un resumen de los principales tópicos son presentados a continuación.

¿Cuál es su opinión frente a las alternativas "Matemática pura" y "Matemática aplicada"?

Primero debo aclarar que me siento matemático, de la matemática aplicada. Pienso que la matemática pura es una ciencia muy vieja y casi todas las cosas importantes fueron encontradas hasta el siglo pasado. Se dice que la matemática pura es más profunda o que la matemática aplicada es más superficial, pero siempre habrá una cierta lucha entre ambas ramas.

Aunque el desarrollo de la matemática apunta hacia la matemática aplicada, es claro que la matemática pura debe tener un muy buen nivel en las universidades y que debe entregarse, también es muy buen nivel, en los primeros años universitarios.

La mayoría de los matemáticos no se interesan por los temas de computación. Son estáticos por cuanto prefieren temas tradicionales en vez de los métodos de la computación.

De los 1500 funcionarios que estaban en el centro de computación alemán en que trabajé, 1000 se dedicaban a problemas estadísticos (generados por modelos economicos), el resto a problemas de origen técnicos, siendo só1050 los que trabajaban en análisis numérico. 
¿Qué opina acerca del análisis numérico y la optimización en Chile?

Tengo la impresión que en análisis numérico se trabaja relativamente poco pero en muy buen nivel. Por ejemplo el proyecto "La ecuación de Poisson" (en la Universidad del Norte) utiliza conceptos muy modernos.

Respecto a optimización, personalmente me intereso en el análisis funcional no lineal (Ecuaciones diferenciales parciales no lineales). En la Universidad de Chile se ha desarrollado un programa muy profundo en optimización. Tengo una muy buena impresión de lo que allá se hace y creo que en este momento pueden competir con cualquier universidad del mundo.

¿ Qué diferencia del funcionamiento vé Ud, entre los sistemas universitarios alemán y chileno, referente a la matemática ?

Alemania es pobre en recursos naturales y por tal razón debe efectuar mucha investigación, cuyos resultados le permiten hasta exportar tecnología. Vivimos de nuestra investigación y por eso hay tanta libertad y autonomía de mandos en las universidades.

En las universidades hay dos tipos de profesores, los "funcionarios" y los "empleados". Los primeros son cargos vitalicios y tienen cuatro niveles jerárquicos aún cuando uno de ellos sólo existe formalmente. Estos profesores hacen 4 horas semanales de clases y un seminario de 2 horas en que exponen sólo los estudiantes. El resto del tiempo es de libre disposición ya que las tareas administrativas son evitadas por los profesores con el fin de invertir el máximo 
de tiempo a la investigación. Las vacaciones (5.5 meses al año) son normalmente dedicadas a intensificar sus investigaciones lo que es altamente provechoso para Alemania. Los profesores "empleados" estatales generalmente no trabajan toda su vida en la universidad y dictan sus cursos a nivel de ingreso. Estan a cargo de trabajos con muchos ejercicios y normalmente hacen 10 horas semanales de docencia.

Respecto a la docencia matemática en la universidad, es conveniente notar que no hay un proceso de selección entre la enseñanza media y la universidad. Los cursos básicos son modulares y sirven indistintamente para todas las carreras, existiendo sólo un curso por cada materia y a cargo de sólo un profesor (normalmente son cursos de 700 alumnos). E1 alumno debe resolver listas semanales de ejercicios y al obtener cierto procentaje de problemas correctos tiene derecho a rendir una prueba. Aprobada esa prueba recibe un certificado y al tenerlos en número suficiente, puede rendir un examen después de 3 ó 4 semestres. Aprobado tal examen son muy escasos los alumnos que dejan inconclusos sus estudios $y$ normalmente aprueban su examen final con muy buena nota. Para sobrevivir los cuatro primeros semestres es necesario trabajar con una carga promedio de $60 \mathrm{hrs}$. semanales. Este fuerte régimen de estudios produce un rendimiento muy bajo en el primer nivel. El alumno asiste a 12 horas de matemáticas, 6 de otra ciencia pero ya a la altura del tercer semestre se ha retirado e $60 \%$ de los alumnos. Entre los estudiantes de ingeniería este porcentaje está entre el 70 y 75 . Si el porcentaje de éxito fuera del $50 \%$ o mayor, existe precupación y se piensa que los contenidos no fueron suficientemente difíciles. 